\title{
A Belmont Report for Animals?
}

\author{
HOPE FERDOWSIAN, L. SYD M JOHNSON, JANE JOHNSON, ANDREW FENTON, \\ ADAM SHRIVER, AND JOHN GLUCK
}

\begin{abstract}
Human and animal research both operate within established standards. In the United States, criticism of the human research environment and recorded abuses of human research subjects served as the impetus for the establishment of the National Commission for the Protection of Human Subjects of Biomedical and Behavioral Research, and the resulting Belmont Report. The Belmont Report established key ethical principles to which human research should adhere: respect for autonomy, obligations to beneficence and justice, and special protections for vulnerable individuals and populations. While current guidelines appropriately aim to protect the individual interests of human participants in research, no similar, comprehensive, and principled effort has addressed the use of (nonhuman) animals in research. Although published policies regarding animal research provide relevant regulatory guidance, the lack of a fundamental effort to explore the ethical issues and principles that should guide decisions about the potential use of animals in research has led to unclear and disparate policies. Here, we explore how the ethical principles outlined in the Belmont Report could be applied consistently to animals. We describe how concepts such as respect for autonomy and obligations to beneficence and justice could be applied to animals, as well as how animals are entitled to special protections as a result of their vulnerability.
\end{abstract}

The year 2019 commemorates the fortieth anniversary of the Belmont Report. As a foundational document in research ethics, it establishes the principles to which human research should adhere: respect for persons (generally articulated as respect for autonomy), obligations to beneficence and justice, and special protections for vulnerable individuals and populations. These principles then provide a base from which actionable and robust operational requirements-namely, informed consent, assessment of risks and benefits, and selection of subjects rationally flow. In the United States, the Belmont Report is an essential reference for institutional review boards that evaluate human research proposals. The principles outlined in the report serve to protect potential human research subjects, including those with compromised capacity to provide informed consent. Drawing on the work of the National Commission for the Protection of Human Subjects of Biomedical and Behavioral Research, which authored the Belmont Report, many federal agencies and private and public institutions have adopted a uniform set of regulations known as the "Common Rule," which offers specific protections for children, incarcerated individuals, and pregnant women and their fetuses. ${ }^{1}$

The development and publication of the Belmont Report followed concerns about human research, including examples detailed in Henry Beecher's 1966 paper "Ethics and Clinical Research," which revealed that human research was frequently conducted with insufficient attention to informed consent, risks to participants, or sound ethical justification. ${ }^{2}$ By 1972, news reports exposed concerns about the fortyyear-long U.S. Public Health Service Syphilis Study at Tuskegee. Researchers did not obtain informed consent from the African American men targeted for inclusion in

Adam Shriver's work on this project is supported by Wellcome Trust grant 203132/Z/16/Z.

The section title was incorrectly listed as "Guest Editorial" in the original online version of this article. This will be corrected in print and an erratum will be published. 
the study, and the men were not notified of the availability of penicillin when it became the established effective treatment regimen for syphilis in 1947. Many of the men were impoverished, with limited access to healthcare, education, and other resources. In response to public concerns, a nine-person advisory panel was established, which determined the study was ethically unjustified. Within five months of the panel's appointment, the Tuskegee Syphilis Study was halted. ${ }^{3}$

Subsequently, public concerns about unethical research practices grew. Initially, these critiques led to minor changes in National Institutes of Health (NIH) oversight requirements. However, recognizing the need for broader guidance, the U.S. Congress soon authorized the establishment of the National Commission for the Protection of Human Subjects of Biomedical and Behavioral Research. Four years later, the Commission, which was composed of researchers, philosophers, and public representatives, produced the Belmont Report. Today, the report's reach and influence extends around the globe.

\section{The Situation for Nonhuman Animals}

The same year Henry Beecher published his groundbreaking article in the New England Journal of Medicine, the U.S. Congress passed the Animal Welfare Act. ${ }^{4}$ However, the Animal Welfare Act and subsequent laws and policies governing animal research in the U.S. have done relatively little to address ethical problems within research involving nonhuman animals (hereafter: "animals"), including which animals are covered by various policies, and how decisions about their treatment should be made. ${ }^{5}$ Though the original intent of the Animal Welfare Act was to prevent the unauthorized acquisition of some dogs and cats for research purposes, subsequent amendments have expanded or restricted the scope of the Act. In 2002, the majority of animals used in research (birds, rats of the genus Rattus, mice of the genus Mus, fish, and farm animals) were specifically excluded from the Act, due in part to the lobbying efforts of industries that profit from their extensive use. ${ }^{6}$ In contrast, other federal research guidelines, such as the Guide for the Care and Use of Laboratory Animals, cover all vertebrates. ${ }^{7}$

Today, there continue to be significant inconsistencies among animal research regulations, and individuals serving on institutional review committees still have no clear set of ethical principles from which to ground decisions about protocol approval. Whereas ethical concerns about human research led to a government request for guiding principles for human research, ethical concerns about animal research have been addressed on a piecemeal basis.

Today, biomedical ethical principles including respect for autonomy, nonmaleficence, beneficence, and justice are widely recognized and applied within human medicine and research. ${ }^{8}$ However, the situation genuinely differs for decisions about the use and treatment of animals within research.

Currently, researchers, reviewers, and oversight bodies generally rely on the "3Rs" framework first proposed by William Russell and Rex Burch almost sixty years ago. ${ }^{9}$ This framework, which restricted its focus to vertebrate animals, originally emphasized replacement of "sentient " animals with so-called " less sentient" animals or nonanimal research methods, reduction in the numbers of animals used in particular experiments or studies, and refinement of experimental techniques to reduce stress or distress. Since then, different jurisdictions have expanded the meaning of animals (e.g., the inclusion of invertebrates like cephalopods), ${ }^{10}$ 
replacement (e.g., the replacement of all animals), ${ }^{11}$ reduction (e.g., a reduction of the number of animals used in a study to what is considered minimally necessary for adequate statistical power), ${ }^{12}$ and refinement (e.g., attention to general wellbeing rather than just reduction in procedure-generated stress or distress). ${ }^{13}$ However, these changes have not occurred universally, and the 3Rs framework still does not reflect a set of principles by which to judge the ethical permissibility of proposed research conduct. ${ }^{14}$ The 3 Rs framework assumes the necessity of animal research, reflecting the prevailing "science first" attitude outlined by Russell and Burch, as well as a general presumption that, when in conflict, human interests outweigh animal interests.

Recently, there have been limited changes in animal research policy, reflecting shifts in attitudes, and notably, a critical reexamination of the presumption that all research with animals is justified by benefits for humans. For example, following substantial public controversy and a 2010 Congressional request, the NIH commissioned the formation of an Institute of Medicine (IOM) Committee on the Use of Chimpanzees in Biomedical and Behavioral Research. Although the IOM Committee was asked to offer guidance only on the scientific necessity of chimpanzees in biomedical and behavioral research, it determined that assessment of the necessity of chimpanzee research could not fully avoid ethical considerations. Though its report left numerous moral questions about animal research unanswered, ${ }^{15}$ the Committee concluded that chimpanzees are largely unnecessary to ongoing and future research endeavors. ${ }^{16}$ By 2013, NIH phased out all chimpanzee research protocols that did not satisfy the Committee's principles and corresponding criteria. In November 2015, in part because of changes in the conservation status assigned captive chimpanzees by the U.S. Fish and Wildlife Service, NIH announced it would no longer support biomedical research on chimpanzees. ${ }^{17}$

Public concerns and attention to animal research policy have also extended to other species. In 2018, Senator Cory Booker introduced legislation in the U.S. Senate that would seek to prevent all nonhuman primate research independently considered to be unnecessary or unethical. ${ }^{18}$ Numerous scientists, legal scholars, and other professionals endorsed the legislation. Similarly, as a result of public opposition and Congressional action, maternal deprivation and addiction experiments involving monkeys, fatal experiments on dogs and cats, and some other animal experiments have come under increased scrutiny and, in some cases, these experiments have been halted. ${ }^{19}$ Such changes in policy and practice reflect growing public sentiment. Roughly half of Americans think that medical testing on animals is morally unacceptable, and public opposition to animal research has steadily increased over the past decade. ${ }^{20}$ According to a Gallup Poll, approximately one-third of Americans believe that animals should have the same rights as people to be free from harm and exploitation. ${ }^{21}$

Outside the U.S., there has been similar attention to animal research policy. In 2010, the EU Parliament called for immediate restriction on the use of great apes in research, and for phasing out the use of all nonhuman primates. Numerous countries in Europe and around the world have enacted total or partial bans on the use of some animals in research.22 A number of high profile reports on research with nonhuman primates have been issued internationally in the last decade, including the 2006 UK Weatherall Report ${ }^{23}$ and the European Commission's SCHER report. ${ }^{24}$ However, both of these reports simply presumed the "necessity" of research with nonhuman primates and failed to provide a meaningful and impactful analysis based on established ethical principles. These reports, like others, have neglected the fact that research deemed as necessary raises the need for but does not replace 
ethical considerations or imply that such research is ethical. Furthermore, although some attention in the U.S., Europe, and elsewhere has focused on nonhuman primate and nonprimate species such as dogs and cats, decisions involving other animals also deserve broader and more consistent ethical guidance, given similarities in capacities, vulnerabilities, and corresponding moral questions across species.

\section{Grounds for Extending Human Research Principles to Animals}

To date, no expansive, sustained, and organized effort like that of the National Commission for the Protection of Human Subjects of Biomedical and Behavioral Research has addressed the use of animals in research. No document regarding animal research has approximated the scope and influence of the Belmont Report despite advancements in our understanding of animals' emotional and intellectual capacities. ${ }^{25}$ As described elsewhere, animal experimentation is arguably plagued by many moral problems analogous to those Henry Beecher described: that is, issues related to consent, harm, and lack of benefit to the experimental subject. ${ }^{26}$

Within human populations, there are significant differences in mental capacity, moral aptitude, and other qualities that have been used historically to justify differences in the presumed moral status and treatment of humans and other animals. As other authors have argued, no compelling argument for this view has been identified. ${ }^{27}$ Nonetheless, there is a universal expectation that humans with unequal gradations of these qualities will be treated in accordance with the same basic underlying principles that are broadly recognized within biomedical ethics, as well as in other areas of society. ${ }^{28}$ Using widely recognized and accepted principles to guide research decisions offers a distinct advantage over other approaches. Understood as fundamental moral commitments, principles serve as a foundation for reasonable and internally consistent analysis and action, although their specification may differ from one context or situation to another.

The importance of similarly principled changes in animal research ethics is highlighted by reflection on the current state of the foundations of research ethics (writ large to include the scientific use of both humans and other animals). Current standards in research ethics for humans and for animals are significantly different, and this differential treatment exists despite some of the clear and relevant similarities between potential human and animal research subjects. Appeals to anthropocentric speciesism are the most common justification for treating animal subjects differently, but they have consistently failed to stand up to philosophical scrutiny. ${ }^{29}$ Many have scientifically justified the use of animals in research by citing their similarities to humans, while neglecting how the common potential for vulnerability and mental and physical suffering influences ethical justifications. Humans and other animals have interests in self-preservation, living free of unnecessary constraints, and meeting their basic and complex needs. Like humans, other animals are susceptible to having their basic interests discounted. Additionally, characteristics determining quality of life are relevant to the consideration of an individual's interests, regardless of species classification, and qualification for special protections. ${ }^{30}$ Nothing other than ideological bias and serious human fears of death and disease can motivate the consistent prioritizing of human interests over the relevantly similar interests of other animals. These fears and biases do not make for sound ethical judgment. A very basic insight from moral philosophy is that an objective ethic is not compatible with arbitrary distinctions, values, or standards. This, indeed, 
lies at the foundation of philosophical critiques of moral theories like ethical egoism, and such prejudices as ableism, classism, ethnocentrism, heterosexism, racism, and sexism. Similarly, the differential treatment of human and animal research subjects is grounded in arbitrary distinctions, values, and standards. Such differential treatment does not just adversely affect the objectivity of animal research ethics. The edifice of human research ethics is also built on these arbitrary distinctions, values, and standards. Until this situation is appropriately rectified, the objectivity of human and animal research ethics will remain in question.

In light of these issues, here, we assume that justifications for significant differences between human and animal research regulations based on a claim that humans have a higher moral status are mistaken. Accordingly, we assume that animal interests should be given approximately equal moral weight as human interests and therein consider the implications of extending human research principles to animals. We describe how widely recognized concepts within biomedical ethics, such as respect for autonomy and obligations to beneficence and justice (as reflected in the Belmont Report), could be applied to animals, as well as how animals could be entitled to special protections as a result of their vulnerability. ${ }^{31}$

\section{The Principles and Their Applications}

\section{Respect for Persons (Autonomy) and Informed Consent}

Within the Belmont Report, "respect for persons" incorporates two separate but related moral requirements: to acknowledge autonomy and to protect those with diminished autonomy. Though it is possible that the Commission had other intentions in selecting the principle of "respect for persons," 32 its documented intent "requires that subjects, to the degree that they are capable, be given the opportunity to choose what shall or shall not happen to them." 33

Autonomy commonly refers to the capacity for self-determination. It is both a capacity for self-rule, and an ability to protect or further one's own interests. As a result, it is intimately related to vulnerability. Subjects who lack autonomy lack the ability to adequately represent their own interests, which makes them vulnerable to exploitation and harm.

There are many open questions about the degree to which humans and other animals display autonomy or autonomous action. ${ }^{34}$ It is now generally accepted that animals-including chimpanzees, crows, elephants, macaques, orcas, and wolves, among others-form daily goals and order their behavior accordingly. ${ }^{35}$ Alliances or coalitions, displayed by animals such as chimpanzees, dolphins, and others, reflect goals and planned behaviors that extend beyond momentary concerns. Older members of these animal communities behave in ways that satisfy their preferences, which effectively track what they value and their basic interests. ${ }^{36}$ Many animals also provide for their young, indicating that they also track the preferences or interests of animals in their care. ${ }^{37}$ These behaviors and attendant capacities evidence a capacity for self-determination. In humans and animals, the capacity for self-determination can change acutely or chronically over the course of a lifetime as a result of neurological, psychological, and emotional maturation or degeneration, or other changes in aptitude. Nonetheless, selfdetermination, of varying degrees, is essential to wellbeing in humans and many animals, and interference with self-determination can lead to physical and mental 
distress, as well as disorders such as learned helplessness, posttraumatic stress disorder, and depression. ${ }^{38}$

As the Belmont Report indicates, autonomy is also characterized by an ability to make informed decisions free of undue influence. External "circumstances that severely restrict liberty," as in the case of prisoners, can adversely impact one's ability to exercise autonomy. ${ }^{39}$ Sources of fear and other forms of intimidation, such as captivity, can limit the expression of self-determination.

Though it is important to acknowledge capacities like autonomy and their implications, as the Belmont Report points out, it is as important to protect those with diminished autonomy, whether such diminishment is due to factors that are intrinsic or extrinsic to the individual. In such cases, two general rules apply. To the degree they are able, even individuals with compromised autonomy should be provided opportunities to choose what will or will not happen to them-a standard of welfare that is also increasingly recognized as applicable to animals. ${ }^{40}$ However, to the degree that individuals are incapable of making informed decisions, they should be protected from harm.

The application of respect for persons in human research typically takes the form of seeking and securing informed consent before inclusion in a research study. Informed consent requirements generally include three elements: the delivery of sufficient information, comprehension, and voluntariness. ${ }^{41}$ Guidelines provide opportunities for those with diminished capacities to represent their own interests in the decisionmaking process by respecting "the objections of...subjects," 42 allowing individuals to dissent even if they are not deemed to have the capacity to provide informed consent. In some cases, surrogate decisionmakers charged with choosing what is in the potential research subject's best interests may offer permission on the individual's behalf. Regardless of the circumstance, coercion, in which an individual is threatened with harm, and undue influence are prohibited: "Inducements that would ordinarily be acceptable may become undue influences if the subject is especially vulnerable." 43

There remain unanswered empirical and conceptual questions about autonomous capacities in animals. Human-animal interactions, and the crudeness of our understanding of animals, are unlikely to permit meaningful informed consent. Therefore, it is important to consider whether concepts used in human research such as dissent, assent, and surrogate decisionmakers are sufficient for formulating decisions about the use of animals in research.

Within pediatric research ethics, researchers and regulators increasingly recognize that the sustained dissent of even very young children should be respected, unless perhaps the study promises direct benefits that are otherwise unavailable. This requirement is motivated by obligations to avoid harm associated with disrespecting the corresponding level of self-determination of the child. ${ }^{44}$ Elsewhere, requisite capacities needed to express sustained dissent have been described. ${ }^{45}$ Briefly, young children who express sustained dissent (1) have the capacities to experience pain or distress; (2) can anticipate its occurrence; and (3) can express a preference that the pain or distress stop (or not occur). Many animals also possess these capacities, as evidenced by relevantly similar neurological structures, escape or avoidance behaviors, and the expression of behavioral preferences. However, an absence of escape or avoidance behaviors does not necessarily indicate an absence of dissent. ${ }^{46}$ Like children, animals are more likely to adapt or suppress certain behaviors in an attempt to protect themselves from individuals in a position of power or influence-further underscoring their vulnerability. Captive conditions and other practices used during the course 
of laboratory research interfere with animals' capacities to express dissent, deepening their vulnerability to manipulation and coercion. Therefore, in the current context, absence of sustained and recognizable indicators of dissent alone is generally not a sufficient criterion for permitting research with animals to proceed.

There are unresolved questions about whether animals can express assent in ways humans reliably understand. ${ }^{47}$ Assent, compared with dissent, may be considered a more morally rigorous requirement for participation in research or other activities. As an affirmative expression of agreement, assent implies active participation, whereas an absence of dissent can suggest passive or confused participation and therefore less certainty about a willingness to participate. However, in most cases involving animals, it is unlikely that, even in the best of circumstances, they could make an informed decision about the potential risks or benefits of research generated by perceived human needs.

The potential use of surrogate decisionmakers in animal research also presents special difficulties. First and foremost, consent from human surrogate decisionmakers is always suboptimal compared with consent from a capable research subject. However, current regulatory guidelines involving individuals who cannot provide consent (e.g., young children) allow for the input of a surrogate decisionmaker (e.g., at least one parent) who can provide or refuse permission on the child's behalf. In these cases, respect for sustained dissent and assent of the child (if they are capable of providing it) are also required. However, as outlined above, the case of animals raises particular challenges related to dissent and assent. Since convincing arguments for why nonbeneficial research is acceptable in those who cannot provide consent are lacking, a conservative standard is required.

In practice, respect for the autonomy and vulnerability of animals would require respecting their freedom and choices to the extent that they are capable of asserting their decisions, and to the extent we are capable of understanding their choices. At the very least, the following criteria would be required: an absence of punishment, coercion, restraint, or confinement; real options and alternatives appropriate to the individual and their normal circumstances; and surrogates who are not unduly compromised in their capacity to represent the animal's interests by their own personal or professional conflicts of interest. Surrogate decisionmakers should also be capable of making an independent, informed decision free of coercion or undue inducement. If a surrogate cannot reliably understand the cognitive, ethological, and ecological factors required for the health and wellbeing of the individual animal, and impartially represent their individual interests, a cautionary approach would require avoiding enrollment in research.

\section{Beneficence and Assessment of Risks and Benefits}

According to the Belmont Report, the principle of beneficence is interpreted as an obligation. Beneficence is often taken to mean "doing good," but in the Belmont report, the principle is based on two general rules: (1) do no harm (nonmaleficence; a principle believed by many scholars as also embodied in the Hippocratic Oath); and (2) maximize possible benefits while minimizing possible harms. ${ }^{48}$ These rules, interpreted literally, would clearly be in tension, as there is a difference between not harming and merely minimizing harm (or risk of harm). However, in human research, the principle of beneficence is operationalized through a 
requirement that possible risks to participants are minimized and that there is a favorable benefit/risk ratio for participants.

The dual requirement that harms are minimized and that there is a favorable benefit/risk ratio is noteworthy, since either rule by itself would be insufficient. For example, if the only requirement for the principle of beneficence were that the risk of harms be minimized relative to proposed research, some human research could be deemed permissible even when potential harms significantly outweigh potential benefits. Conversely, having only a requirement for a favorable benefit/ risk ratio without an obligation to minimize harms would allow research that included preventable or unjustifiable harms to proceed. Thus, it is only in tandem that these two rules (when properly executed) ensure that undue and preventable harms are avoided.

The existing regulatory situation is quite different for animals. For example, the 3Rs framework utilized by most institutional review committees often leads to claims that animal research does require benefit/risk analysis, since funding sources are expected to assess benefits in their funding decisions and institutional review committees attempt to reduce harms associated with specific protocols. ${ }^{49}$ However, in the absence of a direct comparison of potential harms and benefits, problematic research resulting in significant harms to animals can readily proceed. This problem is particularly exacerbated in exploratory research involving animals, in which purported benefits may only amount to faint promises of discoveries. In practice, harms to animals are afforded comparably little weight. ${ }^{50}$ The situation reflects a problem that the Belmont Report (and subsequent regulatory policies) avoided by including a dual criteria notion of beneficence.

The IOM Committee's finding regarding chimpanzees provides a clear example of the failings of the current approach used in animal research. The Institutional Animal Care and Use Committees (IACUCs) at institutions performing research with chimpanzees completed their official duties in reviewing research protocols, minimizing harms relative to proposed protocols. Nevertheless, most or all of federally funded chimpanzee research was ultimately deemed unnecessary, and presumably scientifically and ethically unjustified, by the IOM Committee. In this case and others, the absence of a direct comparison of potential harms and benefits, or a pro forma exercise to meet review committee expectations, would continue to permit ethically problematic research.

Though duties of nonmaleficence and beneficence are alluded to in some animal research guidelines, current provisions do not afford sufficient attention to these principles. For example, the most recent edition of the Guide for the Care and Use of Laboratory Animals requires that, for certain research, "the IACUC is obliged to weigh the objectives of the study against potential animal welfare concerns." 51 Recently, American and European organizations overseeing the use of animals in research released a two-part set of recommendations for benefit/risk analysis in animal research. ${ }^{52}$ However, as noted by Anna Olsson and colleagues, the report "has no legal status and is less than clear on a number of points." 53 Individuals responsible for reviewing protocols involving animals still have no clear framework by which to prohibit research with an unfavorable benefit/risk profile. As a consequence, full rejection of a protocol is rare; instead, protocol review commonly focuses on modifications along the lines of the $3 R$ s that may still fail to create a favorable benefit/risk profile. Sincere attention to the principle of beneficence in animal research would, in contrast, necessarily include: (1) a full assessment to 
the extent possible of actual and potential harms; (2) a full assessment of potential benefits; and (3) an approach that allows for a transparent and rigorous direct comparison of the risk of harms and potential benefits, along with conditions on what benefit/risk profiles are acceptable.

Within human research, the principle of beneficence requires that the nature and scope of physical, psychological, social, and other harms be considered. However, consideration of a full scope of harms is far less common or perhaps even possible in the general practice of animal research. If considered, risks associated with animal research would include, but not be limited to: harms associated with breeding and transport; separation from parents, siblings, and other conspecifics; an inability to control access to that which meets one's own basic needs; a lack of safety and security; thwarting of an animal's preferences; an inability to fulfill one's full developmental potential; deprivation of a normal, species-appropriate, natural environment; and being killed. ${ }^{54}$

In particular, confinement and captivity in themselves introduce significant risk. ${ }^{55}$ Certain forms of captivity are inherently harmful to animals because these conditions deprive them of ethologically appropriate environments and activities, of liberty and movement, and of basic psychological and social needs, including stimulation, play, and family. Some forms of captivity also impose experiential harms, including stress, boredom, pain, fear, and loss. Currently, within the practice of animal research, the risk — that is, the magnitude and probability —of all of the aforementioned harms is often severe and certain. ${ }^{56}$

Within human research, measured risks and benefits may also include those that affect "the families of the individual subjects, and society at large (or special groups of subjects in society)." 57 Similarly, for animals, in addition to the harms to prospective subjects, other potential harms should be considered. For instance, in the case of free-ranging animals or descendants of 'wild-caught' animals, the direct environmental costs as well as the indirect environmental costs of supporting these practices should be included in any risk assessment.

In contrast to much research on humans, it is rare for justifications offered for animal research to be focused on benefits to individual animals included in experiments. ${ }^{58}$ No clear moral justification for this position is offered in existing laws, policies, or guidelines, though nonspecific, perceived future benefits to humans are often cited as a rationale for the use of animals in research. As in the case of human research, it is important for institutional review committees to ensure research proposals do not exaggerate potential benefits of research. Though it is impossible to predict the potential value of various scientific discoveries, the possibility of unexpected future benefits also exists for research involving humans. Nonetheless, such uncertainty has not prevented ethics committees from requiring a realistic assessment of potential benefits as part of the approval process for human research proposals. Similarly, uncertainty should not be an impediment to conducting a realistic assessment of potential benefits (or risks) for research involving animals.

The Belmont Report also goes farther, suggesting "the assessment of risks and benefits requires a careful arrayal of relevant data, including, in some cases, alternative ways of obtaining the benefits sought in the research." ${ }^{59}$ Even if research has in the past resulted in a particular benefit such as an advancement of knowledge, it is in many cases possible that the benefit could have been achieved through some other less harmful means. It is similarly important for an assessment of benefits 
of research on animals to accurately reflect whether such benefits could only be procured through the proposed research, or whether anticipated benefits could be achieved via alternative methods now or in the future. ${ }^{60}$

Identifying an appropriate approach for a rigorous comparison of potential harms and benefits is arguably the most conceptually challenging aspect of benefit/ risk analyses. Even in the case of inter-human comparisons, there is rarely agreement about how to weigh particular risks against particular potential benefits. For individuals who can provide consent, individual differences influence a willingness, or lack thereof, to undergo specific risks in anticipation of potential benefits. The case is made more difficult when an individual is unable to provide consent. Nonetheless, even when conflicts occur between commitments to the production of societal benefits and risks of harms to individuals, human subjects are commonly protected from serious harms including severe pain, psychological distress, permanent disability, suffering, death, and prolonged captivity-factors which are commonly experienced by many animals used in research. A commitment to nonmaleficence would require that animals be similarly protected from serious harm. However, in current practice, potential benefits to humans, and even the scientific question itself, are given much more weight than known or potential harms to animals. As other authors have argued, a principle of equal consideration of interests would require that harms to animals should be weighed equivalently to relevantly similar harms in humans. ${ }^{61}$ Likewise, potential harms to humans from ungeneralizable or misleading findings should also be included in a full benefit/risk assessment. ${ }^{62}$

\section{Justice and the Selection of Subjects}

"Who ought to receive the benefits of research and bear its burdens?"

This is the question of justice posed by the Belmont Report. ${ }^{63}$ Attention to justice emerged as a result of perceived injustices associated with the systematic selection of individuals for research because of their easy availability, socioeconomic status, compromised position, or manipulability. For example, in the nineteenth and twentieth century, economically disadvantaged patients seeking healthcare within public institutions bore the burdens of research, whereas wealthier private patients reaped its benefits. Later in the twentieth century, researchers systematically targeted prisoners in Nazi concentration camps, African American men living in Tuskegee, Alabama, the Havasupai of the Grand Canyon, hospitalized children with cognitive disabilities, and other vulnerable or exploited populations. ${ }^{64}$ Though the harms imposed on these disparate populations ranged from deceit to torture, each abuse has contributed to an historical background for how "conceptions of justice are relevant to research involving human subjects." 65

Consideration of the principle of justice within the Belmont Report has given rise to moral requirements for fair procedures and outcomes in the selection of subjects, including attention to social justice:

\footnotetext{
"It can be considered a matter of social justice that there is an order of preference in the selection of classes of subjects (e.g., adults before children) and that some classes of potential subjects (e.g., the institutionalized mentally infirm or prisoners) may be involved as research subjects, if at all, only on certain conditions." 66
} 
Generally, individuals, especially those with impaired autonomy, should not be subjected to risks disproportionate to the benefits of the research, and if individuals are members of groups unlikely to benefit from the research, such individuals may need to be excluded from research altogether. ${ }^{67}$

Currently, animals are deliberately and commonly chosen for research as a result of their easy availability and manipulability, as well as institutional and cultural biases in society. Like human populations who were historically and systematically targeted, animals also have a "frequently compromised capacity for free consent." 68 Animals overwhelmingly bear the burdens of research without accessing the benefits. Though vulnerable human populations, including children and prisoners, are protected as a result of their compromised abilities to provide informed consent, animals are currently excluded from similar protections despite possessing similar vulnerabilities.

The concept of vulnerability is widely used but not well-defined in ethical guidelines. The Belmont Report, like many other statements, guidelines, and codes for research ethics, does not define vulnerability. It lists some of the groups or individuals who can be considered vulnerable subjects, including "racial minorities, the economically disadvantaged, the very sick, and the institutionalized," 69 all of whom, historically, were frequently exploited because they were oppressed, easy to access, powerless, or had no one to advocate for their rights and protection. The Common Rule also lists prisoners, children, and pregnant women and their fetuses as groups requiring special protections.

The Council for International Organizations of Medical Sciences (CIOMS) provides perhaps the most comprehensive definition of vulnerability to be found in research codes. ${ }^{70}$ Vulnerability is defined as either (1) lacking the traits of an autonomous person (the incapacity to protect one's own interests, i.e., vulnerability is defined as intrinsic to an individual), or (2) being unable to act autonomously due to constraints on one's freedom to act (the inability to protect one's own interests, i.e., vulnerability is defined as the result of extrinsic conditions). A previous iteration of the CIOMS guidelines stated that "vulnerable persons are those who are relatively (or absolutely) incapable of protecting their own interests. More formally, they may have insufficient power, intelligence, education, resources, strength, or other needed attributes to protect their own interests."71

Under this substantive understanding of vulnerability, it is clear how both children and prisoners are vulnerable. The former group is primarily vulnerable because they fundamentally lack the ability to comprehend and consent to research, or to advocate for their own interests, and the latter is primarily vulnerable because the very conditions of captivity and institutionalization produce external constraints on their autonomy and ability to make free, uncoerced choices and protect their own interests.

Recent work attempting to better understand the concept of vulnerability, as it applies to humans in research and beyond, can be used to further demonstrate how animals are vulnerable subjects in the context of research. ${ }^{72}$ Catriona Mackenzie, Wendy Rogers, and Susan Dodds have developed a taxonomy that captures the variety of ways in which the concept of vulnerability is deployed. ${ }^{73}$ They identify three types of vulnerability: inherent, situational, and pathogenic. Inherent vulnerabilities are intrinsic features of existence to which humans and animals are susceptible as biological creatures (e.g., illness, disease, accidental injury, pain, suffering, and death), while situational vulnerabilities are extrinsic and arise 
from the context or situation in which individuals or groups find themselves. Pathogenic vulnerabilities form a subset of situational vulnerability and can be brought about either through morally dysfunctional relationships, or when attempts to remedy existing vulnerabilities instead worsen them.

Animals in research are vulnerable in all of these ways. As biological creatures they are inherently or intrinsically vulnerable; their embodiment, basic survival needs, and more complex needs endow a susceptibility to harm that can be realized during the course of research. As in the case of humans, animals' experiences matter to their wellbeing. Since many animals have complex needs and are susceptible to suffering if their needs aren't met, a just approach necessitates that their fundamental needs are treated as similar to those of humans-with full and equal consideration. Treating these animals otherwise, simply because of their easy availability and convenience, undermines obligations to justice. ${ }^{74}$

The treatment of animals in society, including in the current research environment, also exposes animals to situational vulnerability. Even animals with the potential to develop the cognitive capacities necessary to make autonomous decisions and protect their own interests are typically deprived of these capacities in captivity, for the same reasons that involuntarily captive and institutionalized humans lack them: the conditions of captivity act as an external constraint on their abilities to act to protect their own interests. Such constraints include their complete dependence on their captors, as well as the use of coercion and inducements to attain compliance or cooperation.

Animals are also pathogenically vulnerable. Motivations for including them in research reveal at least one source of their pathogenic vulnerability. Animals are often selected as research subjects because of asymmetrical power relations, dependency, and species-based discrimination. Human institutions systematically embed a failure to recognize many animals as entities with intrinsic value, instead treating them as expendable instruments for human convenience. The increasing use of genetically modified animals in research is a particularly profound example of pathogenic vulnerability. Humans develop and breed these genetically modified individuals in an attempt to replicate or simulate a human medical condition, with the intention of subjecting them to the harms of research.

In light of the dependent position of many animals in society, their intrinsic and extrinsic vulnerabilities, and an inability to provide informed consent, such animals should be treated as vulnerable subjects. Within human research, vulnerable subjects typically cannot be exposed to risks exceeding those encountered in normal daily life in safe environments (i.e., minimal risk) or a minor increase over minimal risk, particularly when there is no direct benefit to the individual. The Belmont Report does not provide direct ethical guidance for nontherapeutic research with vulnerable subjects, such as children, that exceeds a minimal risk standard. ${ }^{75}$ The Common Rule restricts such research, but does not prohibit it so long as it is likely to result in generalizable knowledge about a "serious problem affecting the health or welfare" of the individual's "group" (in the case of a child, the group would be children with the condition targeted by the research). ${ }^{76}$ Studies that involve more than a minor increase over minimal risk, with no prospect of direct benefit for the individual who cannot provide consent, must be referred to the federal government, which can convene an expert panel to review the research proposal. If approved, such studies typically require multiple permissions, including the permission of 
each parent and the assent of the child in the case of research involving children. Some have proposed that a similar system could be invoked for animals. ${ }^{77}$ However, such approaches may not be morally sufficient to guide decisions about the use of animals in research.

Animals are in some ways more vulnerable, as individuals and as a class, than human populations who cannot provide informed consent. For example, whereas a parent or guardian who represents a child's best interests can provide permission on their behalf (sometimes with a child's assent), it is much more difficult for a human to represent the interests of an animal, since many humans seem not to understand the needs, desires, or interests of other animals as well as they can (presumably) understand other humans. ${ }^{78}$ There are possible exceptions to this, such as companion animals, whom conscientious guardians may know well. ${ }^{79}$ However, generally, these differences suggest that the risk threshold for research involving animals should be as low as, or lower than, the risk threshold mandated for vulnerable human populations, including children. ${ }^{80}$ It would also suggest a need for greater certainty that the risk threshold is not exceeded. A cautious view would require that if animals cannot consent or assent, almost no form of known harm-particularly serious harm-is acceptable. Permissible research might include that which might provide a direct benefit to the individual (with a favorable benefit/risk profile), in the absence of dissent, as well as permission from an appropriate surrogate decisionmaker. As with surrogate decisionmakers for humans, knowing and understanding an individual's interests, needs, and preferences is necessary but not sufficient to represent that individual's best interests.

\section{A Way Forward}

The principles outlined in the Belmont Report do not operate in isolation but in concert. Each represents a crucial dimension of the moral landscape of human research. Here, we have suggested how these enduring principles together could be applied to decisions about the use of animals in research, which-like human research-has historically been plagued by inconsistencies and moral controversy.

Extending and adapting the principles outlined in the Belmont Report to animals would take into account animals' preferences, interests, and vulnerabilities, and therefore have a significant impact on the use of many animals in research, just as a similar approach has influenced human research. Acknowledging the vulnerability of some human research subjects, and protecting them from exploitation, has had a significant impact on the permissibility of research with some human populations. It is difficult to predict what the full effects of acknowledging and acting in accordance with the vulnerability of animals might be, but as is true in the case of vulnerable humans, the possibility of producing undesirable consequences for the conduct of research does not by itself end the moral debate. Indeed, restrictions on research with vulnerable humans has forced researchers to find other ways of doing research with consenting subjects, and further restrictions on animal research can be expected to force change as well. However, it is a logical fallacy to argue that a claim is false or implausible because of its undesirable consequences. As we have indicated, there is ample evidence that many animals are vulnerable in the same ways that some humans are vulnerable. If we are to be 
morally consistent, those similarities will matter, and figure into any calculations about the moral permissibility of using animals in research. As in the case of humans, it is also important to address the very factors that lead to situational and pathogenic vulnerability in the first place.

One way forward might involve reconceptualizing and restructuring animal research to be more akin to human clinical research. Such an approach involves 'animal patients,' who may be considered 'natural' models for human disease since they spontaneously develop a condition or disease analogous to that found in humans. ${ }^{81}$ Rather than being genetically modified or bred for the sole purpose of experimentation, and housed in laboratory conditions, animal patients live in homes with noninstitutional human caregivers and guardians. They can be enrolled into a clinical trial in a way that is analogous to the way human patients are enrolled into multicenter clinical trials. That is, when animal patients present to a veterinarian and are diagnosed with a condition or disease of interest for which experimental subjects are being sought, they could be offered participation in the research. If the animal's guardian consents to their becoming a research participant, they could remain in the guardian's care, and depending on the nature of the research, they could continue to reside in their own home environment while participating in the research. ${ }^{82}$ The requirements for surrogate consent in this type of research would be similar to those designed to protect children and other vulnerable persons, the most important being that the consent of a guardian alone would not be enough to justify the subject's inclusion in research. The research would also need to satisfy other ethical conditions, including the minimization of risk and ensuring a favorable benefit/risk ratio for the patient- that is, the actual animal patient, and not hypothetical humans, would have to enjoy some benefits (or minimally, likely benefits) in exchange for bearing the risks and burdens of the research. A surrogate should also be able to withdraw consent at any time during the relevant study.

The ethical benefits of such an approach include a substantial shift in the balance of burdens to benefits for animals involved in research. Individual animals could benefit by receiving a potentially effective experimental treatment (if potential harms are minimized); animals involved in research would no longer be routinely killed; otherwise healthy animals would not be exposed to alien diseases; and nonhuman species could also benefit from enhanced veterinary knowledge about treating naturally occurring diseases. Additionally, the many burdens, vulnerabilities, and sources of experimental confounding imposed by captivity and confinement would be significantly mitigated.

Other approaches could also align with the principles outlined in the Belmont Report. These could include noninvasive, observational research on animals in their natural environments and data collection by noninvasive means in natural environments, the use of tissue stored in tissue banks, and other data and materials collected for therapeutic purposes. Additionally, efforts could be made to increase participation in research by humans with the capacity to provide informed consent. Such efforts would better align with the concept of social justice outlined in the Belmont Report; specifically, that there should be an order of preference in the selection of classes of subjects that is protective of the most vulnerable members of society. Finally, research priorities and funding should favor innovation that does not include animals or other individuals who cannot consent to research. 


\section{Conclusion}

Scientific knowledge about the capacities of animals increasingly demonstrates that they possess ethically significant capacities like autonomy/self-determination, and capacities to experience pain and psychological distress that are relevantly similar to human capacities. We have also argued that the vulnerability of animals in research is similar to the vulnerability of some humans in the context of their use as potential research subjects. Together, the ethically significant capacities of animals and their vulnerability as research subjects warrant more protections from the harms of research than current regulatory schemes and guidelines provide. Nothing less than ethically-grounded, principled guidelines, similar to those found in the Belmont Report, can adequately address the ethical inconsistencies and inadequacies of research with animals.

\section{Notes}

1. HHS Common Rule. 45 C.F.R. $\S 46.111(3)$.

2. Beecher HK. Ethics and clinical research. New England Journal of Medicine 1966;274:1354-60.

3. U.S. Public Health Service Syphilis Study at Tuskegee. Centers for Disease Control and Prevention 2015 Dec 22; available at https:/ / www.cdc.gov / tuskegee/timeline.htm (last accessed 30 Nov 2017).

4. 7 U.S.C. $\S \S 2131-2159$ (1966), as amended.

5. Ferdowsian HR, Gluck JP. The ethical challenges of animal research: Honoring Henry Beecher's approach to moral problems. Cambridge Quarterly of Healthcare Ethics 2015;24(4):391-406.

6. See note 4, 7 U.S.C. $\S \S 2131-2159$ (1966), as amended.

7. National Research Council Committee for the Update of the Guide for the Care and Use of Laboratory Animals. Guide for the Care and Use of Laboratory Animals, 8th ed. Washington, DC: National Academies Press; 2011.

8. Beauchamp TL, Childress JF. Principles of Biomedical Ethics, 7th ed. New York, NY: Oxford University Press; 2012.

9. Russell WMS, Burch RL. The Principles of Humane Experimental Technique. London, UK: Methuen \& Co., Ltd; 1959.

10. National Centre for the Replacement, Refinement, and Reduction of Animals in Research. What Are the 3Rs?; available at https: / / www.nc3rs.org.uk/the-3rs (last accessed 30 Nov 2017).

11. Canadian Council on Animal Care. Three Rs Microsite: About the 3 Rs; available at http://3rs.ccac. ca/en/about/three-rs.html (last accessed 30 Nov 2017).

12. UK Government Home Office. Guidance: Animal Testing and Research; available at https://www. gov.uk/guidance/research-and-testing-using-animals (last accessed 30 Nov 2017).

13. Australian Government National Health and Medical Research Council. Guidelines to Promote the Wellbeing of Animals Used for Scientific Purposes: The Assessment and Alleviation of Pain and Distress in Research Animals; available at https:/ / www.deakin.edu.au/_data/assets/pdf_file/0004/536629/ nhmrc-pain-and-distress-pdf.pdf (last accessed 30 Nov 2017).

14. Houde L, Dumas C. An ethical analysis of the 3Rs. Between the Species 2007:7; available at https:/ / digitalcommons.calpoly.edu/bts/vol13/iss7/.

15. Beauchamp TL, Ferdowsian HR, Gluck J. Where are we in the justification of research involving chimpanzees? Kennedy Institute of Ethics Journal 2012;22:211-42.

16. National Research Council. Chimpanzees in Biomedical and Behavioral Research: Assessing the Necessity. Washington, DC: The National Academies Press; 2011.

17. Collins FS. NIH Will No Longer Support Biomedical Research on Chimpanzees 17 Nov 2015; available at https: / / www.nih.gov/about-nih/who-we-are/nih-director/statements / nih-will-no-longersupport-biomedical-research-chimpanzees (last accessed 30 Nov 2017). See also Reardon S. NIH to retire all research chimpanzees: Fifty animals held in "reserve" by the US government will be sent to sanctuaries. Nature 18 Nov 2015; available at https://www.nature.com/news/nih-to-retire-allresearch-chimpanzees-1.18817 (last accessed 30 Nov 2017).

18. Booker Legislation Seeks to End Unethical and Unnecessary Testing on Primates, Press Release, December 18, 2018; available at https: / / www.booker.senate.gov / ?p=press_release\&id=873 (last accessed 8 Jan 2019). 


\section{Hope Ferdowsian et al.}

19. Grimm D. Decision to end monkey experiments based on finances, not animal rights. Science Magazine 2015 Dec 14; available at https://www.sciencemag.org/news/2015/12/decision-endmonkey-experiments-based-finances-not-animal-rights-nih-says (last accessed 8 Jan 2019).

20. Strauss M. Americans are divided over the use of animals in scientific research. Pew Research 2018 Aug 16; available at http://www.pewresearch.org/fact-tank/2018/08/16/americans-are-dividedover-the-use-of-animals-in-scientific-research/ (last accessed 8 Jan 2019).

21. Riffkin R. In U.S., more say animals should have same rights as people. Gallup 2015 May 18; available at https:/ /news.gallup.com/poll/183275/say-animals-rights-people.aspx (last accessed 8 Jan 2019).

22. CBC News. Safe Haven for Chimps: Canada's Position on Chimpanzees in Research; available at http:// www.cbc.ca/natureofthings/features/using-chimps-for-research-in-canada (last accessed 2 Feb 2018).

23. Weatherall D. The Use of Non-Human Primates in Research: A Working Group Report Chaired by Sir David Weatherall FRS FMedSci 12 Dec 2006; available at https://royalsociety.org/topics-policy/ publications/2006/weatherall-report/ (last accessed 30 Nov 2017).

24. European Commission Scientific Committee on Health and Environmental Risks (SCHER). The Need for Non-Human Primates in Biomedical Research, Production and Testing of Products and Devices 18 May 2017; available at https:/ / ec.europa.eu/health/sites/health/files/scientific_committees / scheer/docs/scheer_o_004.pdf (last accessed 30 Nov 2017).

25. The Sundowner Report represents perhaps the most substantive attempt by a U.S. agency to create ethical principles for animal research. In 1996, it established three principles to guide decisions about the use of animals by the National Aeronautics and Space Administration (NASA): respect for life, societal benefit, and nonmaleficence. However, the report did not offer a clear defense for or specific applications of its principles, including how the interests of individual animals should be weighed in decisions about their inclusion in research. See National Aeronautics and Space Administration. 1996 Principles for the Ethical Care and Use of Animals - Sundowner Report; available at http: / / www.iacuc.ucsf.edu/Links / awSundwnr.asp (last accessed 30 Nov 2017). Outside the U.S., some guidelines have incorporated noteworthy but vague language about "respect for" the "dignity of" animals; however, questions about the interpretation and application of these concepts remain. Furthermore, these principles have not been widely applied to decisions about the use of animals in research. See Australian Government National Health and Medical Research Council. Australian Code for the Care and Use of Animals for Scientific Purposes, 8th ed. (2013); available at https:/ /www. nhmrc.gov.au/guidelines-publications/ea28 (last accessed 30 Nov 2017). See also Schindler S. The animal's dignity in Swiss Animal Welfare Legislation--challenges and opportunities. European Journal of Pharmaceutics and Biopharmaceutics 2013;84(2):251-4.

26. See note 5, Ferdowsian, Gluck 2015;24:391-406.

27. Gruen L. The moral status of animals. Stanford Encyclopedia of Philosophy 2017 Aug 23; available at https:/ / plato.stanford.edu/entries/moral-animal/ (last accessed 8 Jan 2019). See also Singer P. Practical Ethics. Cambridge, UK: Cambridge University Press; 2011. See also Choe Smith CU. Confronting ethical permissibility in animal research: Rejecting a common assumption and extending a principle of justice. Theoretical Medical Bioethics 2014;35:175-85.

28. Ferdowsian H, Choe C. Extending human research protections to non-human animals. In: Corbey R, Lanjouw A. The Politics of Species: Reshaping Our Relationships with Other Animals. Cambridge, UK: Cambridge University Press; 2013, 232-40. See also Ferdowsian H. Phoenix Zones: Where Strength Is Born and Resilience Lives. Chicago, IL: The University of Chicago Press; 2018.

29. Fenton A. Decisional authority and animal research subjects. In: Andrews K, Beck J, eds. The Routledge Handbook of Philosophy of Animal Minds. New York, NY: Routledge; 2018, 475-84.

30. See note 28, Ferdowsian, Choe 2013, 232-40. See also note 28, Ferdowsian 2018.

31. There are noteworthy dissimilarities between the human and animal research environments. The Belmont Report, like the Declaration of Helsinki and Nuremberg Code, focuses on one species: humans. Efforts to create a similar document for all other animals may appear difficult. In our discussion, we foreground interests and vulnerabilities that can reasonably and broadly be ascribed to many animals. This approach allows for anticipated gains in knowledge about other species, as well as corresponding societal changes in how animal research is perceived, and evolving critical ethical discourse.

32. Tom Beauchamp, who drafted the Belmont Report for the Commission, has speculated that the Commission selected "respect for persons" as its first principle to indicate a level of moral status associated with personhood. See Public Responsibility in Medicine and Research. People $\mathcal{E}$ Perspectives: Tom Beauchamp, PhD - (Excerpt) Reflections on "Respect for Persons" 17 Nov 2014; available at 


\section{A Belmont Report for Animals?}

https:/ / www.youtube.com/watch?v=v2hrMWEmQLw (last accessed 30 Nov 2017). There is still no agreed upon list of capacities for personhood favored by contemporary philosophers; see Andrews K. The Animal Mind: An Introduction to the Philosophy of Animal Cognition. New York, NY: Routledge; 2015. Although, many tend to favor capacities like "autonomy, rationality, self-awareness, linguistic competence, sociability, moral agency, and the capacity for intentional action"; see DeGrazia D. Human-animal chimeras: Human dignity, moral status, and species prejudice. Metaphilosophy 2007;38(2-3):309-29. However, a commitment to the personhood (and not just the potential personhood) of toddlers, many neuro-diverse humans as well as humans with neurodegenerative disorders, cautions against strongly favoring any capacities from this list as jointly necessary. Though this opens a door to the personhood of other animals who possess a number of the same capacities, a lack of consensus on the necessary capacities for personhood leaves this, at present, an unresolved issue. It is possible that a principle such as "respect for bodily sovereignty," which summarily includes respect for bodily liberty and integrity, as recognized in human rights law, would have been more appropriate for what the National Commission intended. Such a principle could also be applied to the case of animals.

33. National Commission for the Protection of Human Subjects of Biomedical and Behavioral Research. The Belmont Report 18 April 1979; Washington, DC: US Department of Health, and Human Services; available at https://www.hhs.gov/ohrp/regulations-and-policy/belmont-report/index.html (last accessed 30 Nov 2017). See Belmont Report, Pt. (B)(1).

34. See note 8, Beauchamp, Childress 2012. See also Beauchamp TL, Wobber V. Autonomy in chimpanzees. Theoretical Medicine and Bioethics 2014;35:117-32. See also Pierce J. Animals and autonomy. Psychology Today 10 Mar 2013; available at https://www.psychologytoday.com/blog/ all-dogs-go-heaven/201303/animals-and-autonomy (last accessed 30 Nov 2017).

35. de Waal FBM, Tyack PL, eds. Animal Social Complexity: Intelligence, Culture, and Individualized Societies. Cambridge, UK: Harvard University Press; 2003. See also Safina C. Beyond Words: What Animals Think and Feel. New York, NY: Henry Holt and Co.; 2015; and Emery N. Bird Brain: An Exploration of Avian Intelligence. Princeton, NJ: Princeton University Press; 2016.

36. See note 35, de Waal, Tyack 2003.

37. van Noordwijk MA. From maternal investment to lifetime maternal care. In: Mitani JC, Call J, Kappeler PM, Polombit RA, Silk JB, eds. The Evolution of Primate Societies. Chicago, IL: University of Chicago Press; 2012. See also Kuczaj SA II, Winship KA. How do dolphin calves make sense of their world? In: Herzing DL, Johnson CM, eds. Dolphin Communication and Cognition: Past, Present, and Future. Cambridge, UK: The MIT Press; 2015, 201-26.

38. Gregory NG. Physiology and Behavior of Animal Suffering. Oxford, UK: Blackwell Science; 2004. See also McMillan F, ed. Mental Health and Well-Being in Animals, $1^{\text {st }}$ edition. Ames, IA: Blackwell Publishing; 2005. See also Panksepp J. Affective Neuroscience: The Foundations of Human and Animal Emotions. New York, NY: Oxford University Press; 2004.

39. See note 33, National Commission for the Protection of Human Subjects of Biomedical and Behavioral Research 1979, Pt. (B)(1).

40. Perlman JE, Bloomsmith MA, Whittaker MA, McMillan JL, Minier DE, McCowan B. Implementing positive reinforcement animal training programs at primate laboratories. Applied Animal Behaviour Science 2012;137:114-26.

41. Information should include "the research procedure, their purposes, risks and anticipated benefits, alternative procedures (where therapy is involved), and a statement offering the subject the opportunity to ask questions and to withdraw at any time from the research." Information should be adapted to meet the subject's capacities, and this obligation increases as risks to the individual become more serious. See note 33, Belmont Report 1979, Pt. (C)(1).

42. See note 33, National Commission for the Protection of Human Subjects of Biomedical and Behavioral Research 1979, Pt. (C)(1).

43. See note 33, National Commission for the Protection of Human Subjects of Biomedical and Behavioral Research 1979, Pt. (C)(1).

44. Diekema DS. Conducting ethical research in pediatrics: A brief historical overview and review of pediatric regulations. The Journal of Pediatrics 2006;149:S3-11. See also Wendler D. Assent in pediatric research: Theoretical and practical considerations. Journal of Medical Ethics 2006;32:229-34.

45. Fenton A. Can a chimp say "no"? Reenvisioning chimpanzee dissent in harmful research. Cambridge Quarterly of Healthcare Ethics 2014;23(2):130-9.

46. See note 29, Fenton 2018.

47. See note 34, Beauchamp, Wobber 2014. See also Kantin H, Wendler D. Is there a role for assent or dissent in animal research? Cambridge Quarterly of Healthcare Ethics 2015;24(4):459-72. See also note 29, Fenton 2018. 


\section{Hope Ferdowsian et al.}

48. See note 33, National Commission for the Protection of Human Subjects of Biomedical and Behavioral Research 1979, Pt. (B)(2).

49. Bennett AJ. New era for chimpanzee research: Broad implications of chimpanzee research decisions. Developmental Psychobiology 2015;57:279-88.

50. Pound P, Nicol CJ. Retrospective harm benefit analysis of pre-clinical animal research for six treatment interventions. PloS ONE 2018;13:e0193758.

51. See note 7, National Research Council Committee for the Update of the Guide for the Care and Use of Laboratory Animals 2012, at 27.

52. Brønstad A, Newcomer CE, Decelle T, Everitt JI, Guillen J, Laber K. Current concepts of harm-benefit analysis of animal experiments-report from the AALAS-FELASA working group on harm-benefit analysis-part 1. Laboratory Animals 2016;50(1_suppl):1-20. See also Laber K, Newcomer CE, Decelle T, Everitt JI, Guillen J, Brønstad A. Recommendations for addressing harm-benefit analysis and implementation in ethical evaluation-Report from the AALAS-FELASA working group on harmbenefit analysis-part 2. Laboratory Animals 2016;50(1_suppl):21-42.

53. Olsson IAS, Silva SPD, Townend D, Sandøe P. Protecting animals and enabling research in the European Union: An overview of development and implementation of directive 2010/63/EU. ILAR Journal 2016;57(3):347-57.

54. Many applied ethicists understand harm to consist of a setback to interests that an individual would not otherwise experience arising from the actions or omissions of another or a naturally occurring event. When animals are killed, they often experience a setback to their interests as described here. Their death, in such circumstances, constitutes harm.

55. Gruen L, ed. The Ethics of Captivity. Oxford, UK: Oxford University Press; 2014.

56. Many animals are capable of feeling pain and experience the same types of nociceptive and neuropathic pain as humans. They experience pain and discomfort associated with disease (also called "sickness behavior"), which can result in lethargy, depression, anorexia, reduced bodily care and grooming, sleep disturbances, and enhanced sensitivity to pain. See Dantzer R, Kelley KW. Twenty years of research on cytokine-induced sickness behavior. Brain, Behavior, and Immunity 2007;21:153160. Additionally, when deprived of a normal life, many animals can experience mental disorders like anxiety disorders, posttraumatic stress disorder, and mood disorders, such as depression. It is also possible that some animals, as well as some humans, are more vulnerable to suffering because of their inability to make sense of their plight, or to escape, or alter their circumstances. The absence of maturation of certain neurological structures might also affect the capacity for suffering, if associated with less flexibility and more limited coping mechanisms. See Varner GE. Personhood, Ethics, and Animal Cognition: Situating Animals in Hare's Two Level Utilitarianism. Oxford, UK: Oxford University Press; 2012. See also Akhtar S. Animal pain and welfare: Can pain sometimes be worse for them than for us? In: Beauchamp TL, Frey RG, eds. The Oxford Handbook of Animal Ethics. New York: Oxford University Press; 2011:495-518; Ferdowsian HR, Merskin D. Parallels in trauma, pain, distress, and suffering in humans and nonhuman animals. Journal of Trauma and Dissociation 2012;13:448-69; and note 50, Pound, Nicol 2018.

57. See note 33, National Commission for the Protection of Human Subjects of Biomedical and Behavioral Research 1979, Pt. (C)(2).

58. This statement applies even when allowing for current veterinary medical research.

59. See note 33, National Commission for the Protection of Human Subjects of Biomedical and Behavioral Research 1979, Pt. (C)(2).

60. Although the concept of replacement is meant to prompt consideration of alternative research methods, this often does not occur in practice.

61. See note 27, Singer 2011. See also Satz A. Animals as vulnerable subjects: Beyond interest-convergence, hierarchy, and property. Animal Law 2009;16:1-50.

62. See Akhtar A. The flaws and human harms of animal experimentation. Cambridge Quarterly of Healthcare Ethics 2015;24(4):407-19.

63. See note 33, National Commission for the Protection of Human Subjects of Biomedical and Behavioral Research 1979, Pt. (C)(3).

64. See Lederer SE. Subjected to Science. Baltimore, MD: The Johns Hopkins University Press; 1995. See also The Final Report of the Advisory Committee on Human Radiation Experiments. The Human Radiation Experiments. New York, NY: Oxford University Press; 1996.

65. See note 33, National Commission for the Protection of Human Subjects of Biomedical and Behavioral Research 1979, Pt. (B)(3).

66. See note 33, National Commission for the Protection of Human Subjects of Biomedical and Behavioral Research 1979, Pt. (C)(3). 


\section{A Belmont Report for Animals?}

67. See note 33, National Commission for the Protection of Human Subjects of Biomedical and Behavioral Research 1979, Pt. (B)(3).

68. See note 33, National Commission for the Protection of Human Subjects of Biomedical and Behavioral Research 1979, Pt. (B)(3).

69. See note 33, National Commission for the Protection of Human Subjects of Biomedical and Behavioral Research 1979, Pt. (C)(3).

70. Council for the International Organizations of Medical Sciences. International Ethical Guidelines for Health-Related Research Involving Humans. Geneva, Switzerland: Council for the International Organizations of Medical Sciences; 2016, Guideline 15; available at https: / / cioms.ch/wp-content/ uploads /2017/01/WEB-CIOMS-EthicalGuidelines.pdf (last accessed 30 Nov 2017).

71. CIOMS does not include animals within its expansive list of vulnerable subjects. See Council for the International Organizations of Medical Sciences. International Ethical Guidelines for Biomedical Research Involving Human Subjects. Geneva, Switzerland: Council for the International Organizations of Medical Sciences; 2002, Guideline 13; available at https://cioms.ch/shop/product/internationalethical-guidelines-for-biomedical-research-involving-human-subjects-2/ (last accessed 30 Nov 2017). If we take the understanding of vulnerability provided by CIOMS seriously, however, and consider evidence that this kind of vulnerability is not bounded by species, then animals are vulnerable in the same ways that many humans are vulnerable. Importantly, CIOMS applies the concept of vulnerability to individuals, not to groups. Some individuals within less vulnerable groups are still particularly vulnerable. This is an important point with respect to animals. While animals living in their natural environments might have vulnerabilities (to predation, weather, famine, etc.), they are not vulnerable in the way that their captive conspecifics are. A captive animal in a laboratory is (generally) protected from weather and food shortages, but is vulnerable in other ways specific to captivity and use in research-such as pain, death, loss of autonomy and freedom, loss of social group and family, etc.

72. Johnson J. Vulnerable subjects? The case of nonhuman animals in experimentation. Journal of Bioethical Inquiry 2013;10:497-504.

73. Mackenzie C, Rogers W, Dodds S. Introduction: What is vulnerability and why should it matter for moral theory. In: Mackenzie C, Rogers W, Dodds S, eds. Vulnerability: New Essays in Ethics and Feminist Philosophy. New York, NY: Oxford University Press; 2014, at 1-29.

74. Choe Smith C. Confronting ethical permissibility in animal research: Rejecting a common assumption and extending a principle of justice. Theoretical Medicine and Bioethics 2014;35:175-85. See also note 61, Satz 2009.

75. See note 33, National Commission for the Protection of Human Subjects of Biomedical and Behavioral Research 1979, B(2).

76. See note 1,45 CFR $46 \S 46.407$.

77. Wendler D. Should protections for research with humans who cannot consent apply to research with nonhuman primates? Theoretical Medicine and Bioethics 2014;35:157-173.

78. There is a fundamental problem of knowing another mind. See Nagel T. What is it like to be a bat? Philosophical Review 1974;83:435-50. Recent ideas about "embodied cognition" provide further empirical grounding to this philosophical question. See also note 32, Andrews 2016.

79. Some scientists might also fall in this category. For example, Marc Bekoff, Nathan Emery, Jane Goodall, and Tetsuro Matsuzawa, who have observed animals for extended periods, also come to know many of these animals, their individual personalities, desires, and interests well.

80. Ferdowsian HR, Fuentes A. Harms and deprivation of benefits for nonhuman primates in research. Theoretical Medicine and Bioethics 2014;35:143-56.

81. Johnson J, Degeling C. Animals-as-patients: Improving the practice of animal experimentation. Between the Species 2012;15(1):43-58, at 44.

82. Such an approach would frequently resemble the way human research subjects live during their research participation, which may have the added benefit of improving the translatability of research. 